\title{
Evaluating the Impact of Task Demands and Block Resolution on the Effectiveness of Pixel-based Visualization
}

\author{
Rita Borgo, Member, IEEE, Karl Proctor, Min Chen, Member, IEEE, \\ Heike Jänicke, Member, IEEE, Tavi Murray, and lan M. Thornton
}

\begin{abstract}
Pixel-based visualization is a popular method of conveying large amounts of numerical data graphically. Application scenarios include business and finance, bioinformatics and remote sensing. In this work, we examined how the usability of such visual representations varied across different tasks and block resolutions. The main stimuli consisted of temporal pixel-based visualization with a white-red color map, simulating monthly temperature variation over a six-year period. In the first study, we included 5 separate tasks to exert different perceptual loads. We found that performance varied considerably as a function of task, ranging from $75 \%$ correct in low-load tasks to below $40 \%$ in high-load tasks. There was a small but consistent effect of resolution, with the uniform patch improving performance by around $6 \%$ relative to higher block resolution. In the second user study, we focused on a high-load

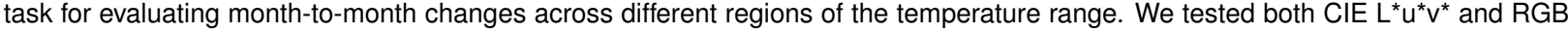
color spaces. We found that the nature of the change-evaluation errors related directly to the distance between the compared regions in the mapped color space. We were able to reduce such errors by using multiple color bands for the same data range. In a final study, we examined more fully the influence of block resolution on performance, and found block resolution had a limited impact on the effectiveness of pixel-based visualization.
\end{abstract}

Index Terms-Pixel-based visualization, evaluation, user study, visual search, change detection.

\section{INTRODUCTION}

Pixel-based visualization is a collection of techniques that use colored position in 2D space to encode data [15]. These techniques can display a large amount of encoded data, and have been found useful in a range of applications, including business and finance [38], bioinformatics [14] and remote sensing [18, 28].

In a typical pixel-based visualization, colored pixels are grouped into blocks (also termed as sub-windows in the literature), and blocks are normally organized in matrix form with two primary attribute dimensions (e.g., month and year). The typical objective of the visualization task is to establish the correlations, causality or other relations between blocks of pixels, and to identify unusual patterns in the data. Block resolution (i.e., the number of pixels in each block) can vary substantially. A block may contain one data value (e.g., temperature), or over a million pixels (e.g., in a satellite image). Visualizing a series of high resolution pixel blocks can benefit from a large power-wall display.

A challenging scientific question naturally arises from such variation: what are the factors that mainly determine user performance with such displays? Will it depend only on the number of pixels in each block? Or will other variables have a greater impact? The answer to such a question will clearly depend on a number of factors, such as the nature of the task, the skill level of the user and, more fundamentally, the limits of human vision, attention and cognition. However, so far, there has been little quantitative analysis of pixel-based visualization, especially in terms of task variations and block variations. It is this gap that we try to fill in the current work.

In three user studies, we examined performance in a common sce-

- R. Borgo and M. Chen are with Computer Science, Swansea University, E-mail: $\{$ r.borgo,m.chen\}@swansea.ac.uk.

- K. Proctor and I. M. Thornton are with Department of Psychology, Swansea University, E-mail: \{cskp,i.m.thornton\}@swansea.ac.uk.

- H. Jänicke is with Interdisciplinary Center for Scientific Computing, Heidelberg University, E-mail: heike.jaenicke@iwr.uni-heidelberg.de.

- T. Murray is with Department of Geography, Swansea University, E-mail:t.murray@swansea.ac.uk.

Manuscript received 31 March 2010; accepted 1 August 2010; posted online 24 October 2010; mailed on 16 October 2010.

For information on obtaining reprints of this article, please send email to:tvcg@computer.org. nario in which month-to-month variations in temperature were visualized over a six-year period. Block resolution was varied within a small range (from uniform patches up to $8 \times 8$ arrays), allowing the whole visual design to be easily reproducible on a ordinary computer displays. In the first study, we examined block resolution and task difficulty, by presenting different comparative visual search and change detection tasks. This initial study allowed us to identify upper and lower limits of performance and to make an initial assessment of the impact of resolution. In the two subsequent studies, we selected tasks at the two extremes of performance, and examined more closely the role of block resolutions and color maps in determining patterns of behavior. Across all three studies, we found that:

- block resolution had a limited impact on the effectiveness of pixel-based visualization;

- task demands and related perceptual constraints accounted for most of the observed variation;

- careful selection of color palettes is essential for reducing taskrelated errors.

\section{Related Work}

Pioneered by Keim [15], pixel-based visualizations are known for the capability of making the best possible use of screen space [17, 10]. Such display can visually present more data than many other techniques, such as iconic and projection-based techniques $[16,6]$. The controls of its design space normally include the choice of color space, subwindow shapes, pixel arrangement, dimension ordering and query specification [27, 19]. With the advent of giga-pixel displays [37], it is desirable to learn how well pixel-based visualization will scale according to the increasing block resolution.

Natural images contain detail at a wide range of spatial scales [29, $32,31]$. The human visual system has evolved mechanisms to parse information according to spatial frequency content $[35,8]$. In image perception, it is thought that coarse-scale information, captured by low spatial frequency filters, conveys information about general shape and structure, while fine-scale information, captured by high spatial frequency filters, carries information about edges and surface texture. Human perception of images at different resolutions has been extensively studied (e.g., [11, 31]). Much work in this area focused on object and face recognition from degraded images. The practical objective, in pixel based visualization, is to achieve cost-effectiveness 


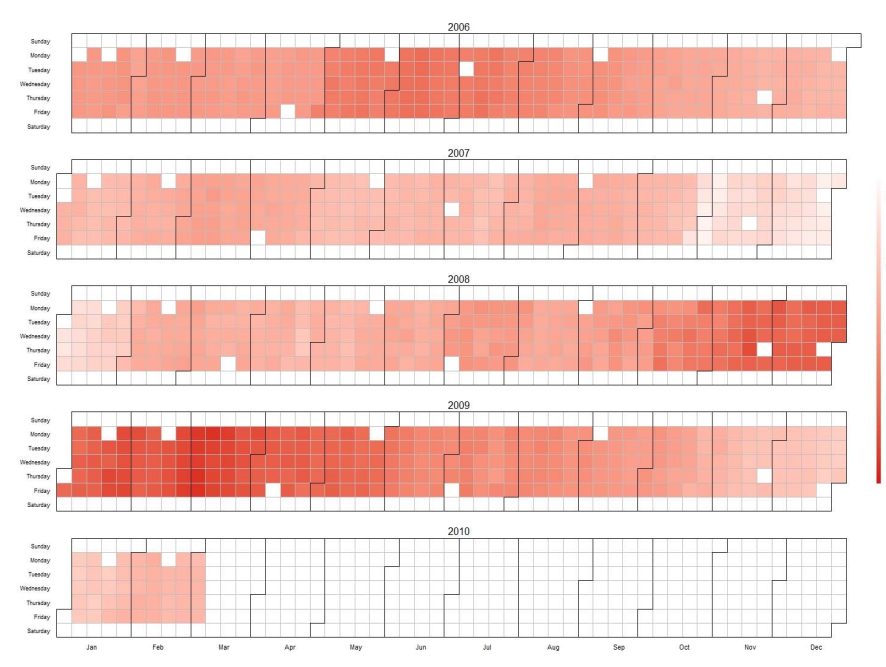

(a) 2006 to date month-year calendar heatmap

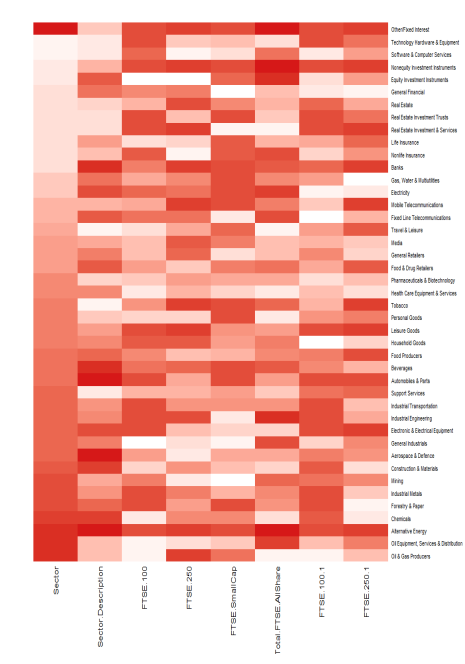

(b) September 2009 heatmap

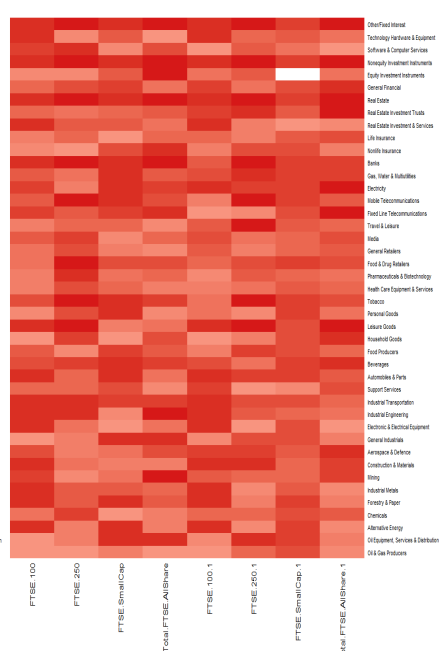

(c) October 2009 heatmap

Fig. 1. (a) A heatmap of Microsoft's stock price adjust close from 2006 to date (data source: Yahoo), generated with $R$ [9] and plotted using Paul Bleicher's calendarHeat function. (b-c) Comparative visualization of two financial heatmaps depicting the secondary market statistics of the London Stock Exchange (courtesy of [7]).

by using relatively low resolution images or videos while maintaining reasonable accuracy and speed in recognition.

In pixel-based visualization, much of the high spatial frequency content originates in the block contours themselves. Indeed, such "block quantization" effects are often used to mask inherent fine-scale information [11]). It remains to be seen whether such local, high frequency "noise" affects the ability of users to extract global, low frequency content. For example, when comparing the average temperatures between two months, will additional details of the daily temperatures help or hinder the decision? However, the practical objective in pixel based visualization is normally the opposite of that for recognition from degraded images. As high-resolution pixel blocks depict more information, it is desirable to use such blocks as long as visualsearch and change detection speed and accuracy rates are reasonable.

In addition to block resolution, human performance with pixelbased visualization may also be influenced by a number of other factors. These may include the effect of surrounding context, working memory and attention demands of a given task. Oliva and Torralba [23] provide and excellent review of the impact of context on human performance. There are many examples in the literature showing that context can influence visual performance (e.g., [3, 5, 13]). Objects appearing in familiar or consistent context (e.g., a plate on top of a kitchen table) will be more efficiently processed than when appearing in an inconsistent context (e.g. on top of a bathtub). This is particularly true when using degraded or low-resolution images (e.g., $[3,31])$. However, most pixel-based visualizations do not benefit from such context, which may affect cognitive load.

Tasks can vary greatly in the demands they place on a user, particularly with respect to memory and attention. Cognitive loads for different types of tasks have been studied in the paradigms of visual search [36], change detection [25], and working memory [24]. For pixel-based visualization, there are no previous studies that relate directly to the cognitive load of different tasks. A primary goal of this paper will thus be to provide an initial assessment of the impact of resolution impact within tasks and their demands in pixel-based displays.

\section{Application Scenario}

Pixel-based visualization can be applied to spatial and non-spatial data. In this work, we consider three application scenarios. The first scenario concerns the use of tabular form of pixel-based visualization to aid the analysis of numeric data captured at regular temporal intervals, such as stock market data. The second scenario concerns the common practice of color coding gene sequences for analyzing individual gene expressions within clusters from a single array. The third scenario concerns the common practice of juxtaposing an image sequence in analyzing remote sensing data.

Fig. 1b-c show a pixel-based visualization depicting the monthly percentages of changes of the 8 indices of the London Stock Exchange between September 2009 and November 2009, for 43 different sectors. The 8 indices are normally grouped into a block, and blocks are organized into year-month matrices. In comparison with Fig. 1a where the average changes are depicted, the $43 \times 6$ pixel blocks in $1 \mathrm{c}$ and $1 \mathrm{~b}$ contain more information useful for localized reasoning while incurring additional perceptual burden in global reasoning. While acknowledging the necessity for the kind of visualization in Fig. 1, it would be helpful to know the effects upon the viewers in their global reasoning. For instance, when reasoning a global trend, would the users mentally determine the average color of each block first? If so, would the errors in determining the average colors result in less accurate reasoning?

Fig. 2a shows a pixel-based visualization of part of a microarray archive derived from a leukemia study [4].

Fig. $2 b$ shows a sequence of Landsat images at two different resolutions with their locations marked on the left. Glaciologists need to compare imagery features at different scales, while trying to establish the overall patterns of the geo-environmental changes. They usually know well the regions that they are interested in. Even when they are given images with a large coverage, their attention immediately focuses on the regions of interests. During a period of collaboration with glaciologists, we observed the typical visual tasks in their scientific discovery work. For instance, they visually estimate the temperature variation over a period, compare the cyclic patterns between different years, and discuss the spatial and temporal relationships between identified phenomena. The varying degrees of uncertainty expressed by the glaciologists when they visualize and analyze such image sequences led us to examine the dependency of the visual analytical accuracy upon the resolution of the pixel blocks and task variations.

There have been numerous prior works on the impact of image resolution upon a viewer's ability to recognize an object or a scene captured by an image (e.g. $[31,33,1])$. These works confirmed that as the resolution increases, viewers perform their recognition tasks better. However, the results of such studies cannot be applied to pixel-based visualization. As the viewers in the above mentioned scenarios perform analytical tasks that are very different from object recognition, the increase of the block resolution could very well have a negative impact. This provides this work with a motivation. 


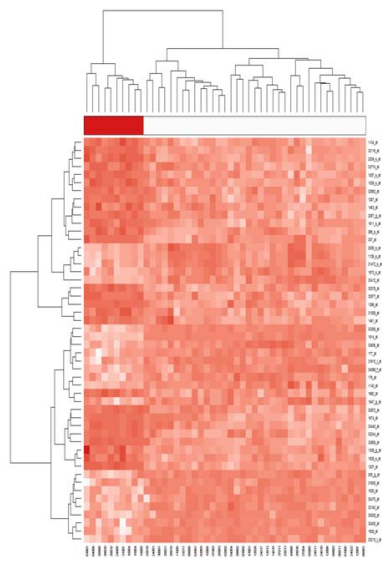

(a)

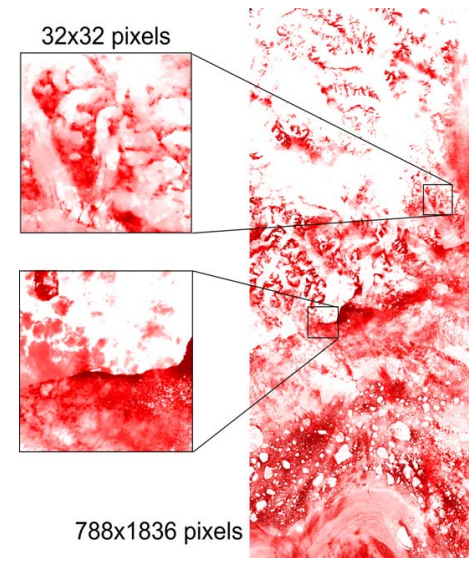

(b)
Fig. 2. (a) A pixel-based bioinformatic visualization, with its spatial layout controlled by two trees; (b) Landsat image of Greenland glacier outlet Geiki.

\section{EXPERIMENTS OVERVIEW}

The three application scenarios mentioned in the previous section indicate several different tasks when using pixel-based visualization. These may include, but not limited to, (i) block-based pattern, change and trend detection (e.g., Figs. 1a and 2a), and (ii) pixel-in-block and pixel-across-blocks analysis (e.g., Fig. 1b-1c and Fig. 2b). The block size can vary from individual primitive blocks (e.g., monthly blocks in Fig. 1b-1c) and composite blocks (e.g., yearly blocks in Fig.1a). In some cases, such as Fig. 2a, the block size varies according to the levels of the two indexing trees. It is thus necessary to make some abstraction from the details of application-specific tasks.

In order to provide our study with an intuitive scenario that all the participants can easily understand, we decided to focus on temporal pixel-based visualization, and chose the common experience of temperature time series as the source of stimuli. A temporal pixel-based visualization is essentially a visualization of multiple time series. In our experiments, we group the values of multiple time series at each time step in a block of pixels.

In designing our experiments, we considered the following factors:

- Data Focuses - Time series data consists of sequences of measurements that follow a specific order. Many time series are expected to exhibit some cyclical behaviors. Such a time series normally features several properties. Amplitude measures the magnitude of the peak of a cycle against the mean of a cycle (or sometimes a predefined base value). Frequency measures the number of cycles in a pre-defined period. Phase shift measures the extent of displacement of one cycle in relation to the preceding cycle, or a predefined reference cycle.

- Task Goals - The goals of time series analysis and pixel-based visualization typically include the measurement of difference and distribution, cycle length at different scales and the identification of peak, trend, seasonality, and irregular fluctuations.

- Types of Changes - There are many types of changes, including existence change (e.g., adding or deleting an object), attribute change (e.g., color, size, etc.), layout change (e.g., relative spatial relationship between objects), and semantic identity change (e.g., a square to a triangle) [26].

- Block Resolution - The number of pixels in each block can vary from application to application. In this work, we explore a relative small range of variation, due to scalability of both stimulus design and test length. This limit is compensated by the varying of block hierarchy.

- Block Hierarchy - Blocks can be the primitive blocks, such as the monthly blocks in Fig. 1b-c and composite blocks such as yearly row in Fig. 1a. More complex hierarchy is exhibited in
$-40$

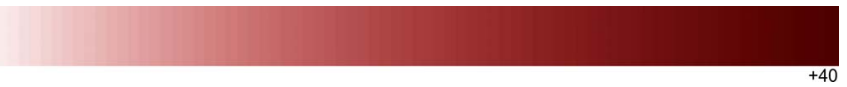

(a) Red White-CIE L*u*v*

$-40$

(b) Red White-sRGB

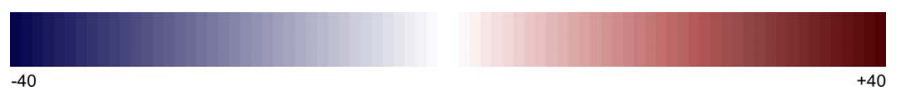

(c) Blue-White-Red Color Scale

Fig. 3. The three color sequences used in experiments 1, 2 and 3.

Fig. 2a, while the hierarchy in Fig. 2b is feature-dependent. In this study, we focus on the basic primitive blocks and composite blocks at one hierarchy upper.

- Colormap - There are many properties of colormaps, including the number of principle colors, and colorimetric transformation. In this work, we had a fixed colormap in the first user study, and examined a small number of foundational variations in the second and third user studies.

All of these factors could influence the perceptual load of a task. We thus designed our first user study, to capture the variation of different tasks. As it is not feasible to explore all combinations of different factors, we designed five tasks to reflect typical tasks in pixel-based visualization for supporting time-series analysis. We found noticeable performance variations between tasks, which likely reflect the different levels of perceptual loads. We then chose two tasks with the best and worst performance for detailed investigation in subsequent studies (studies 2 and 3). Performance was assessed by analyzing both accuracy and reaction time (RT). However, in Studies 1 and 3, RT results were collected as a secondary factor because participants were encouraged to focus on accuracy and were allowed to take as long as they wished to perform the tasks. These RT results are thus prone to a larger variance, and their evidential contribution should be treated with caution. The three studies are described in the following sections.

\section{Color MaPs}

In the choice of our color scales we followed the taxonomy provided in [2] and the ColorBrewer guidelines [12] (in particular for colorblind friendliness). To guarantee a consistent representation of the structure in the data we choose isomorphic colormaps. By design the generated stimuli mimic the output from a weather model computing the variation in relative temperature over a geographic region. The structure of this low spatial-frequency temperature variations over a region, and tasks, which required mental integration of the color-mapped values especially for resolution levels $\geq 1$, made us choose low frequency colormaps therefore guaranteeing a uniform luminance and a monotonically increasing saturation.

\section{USER STUdy 1}

The purpose of this study was to assess accuracy in temperature related judgments as a function of block resolution and specific task demands.

\subsection{Participants}

Twenty four participants ( 9 female, 15 male) took part in this experiment in return for partial course credit or a $£ 5$ book voucher. Students were recruited from the Swansea University community, from a variety of disciplines including Psychology, Humanities, Engineering and Economics. Ages ranged from 18 to $46($ Mean=27.39, SD=5.97). All participants had normal or corrected to normal vision and were not informed about the purpose of the study at the beginning of the session. 
Table 1. User Study 1. Tasks description and relative complexity.

\begin{tabular}{|c|c|c|c|c|c|c|c|}
\hline Task & Question & Unit & Task Goals & Data Focuses & Type of Change & Block Hierarchy & Chance \\
\hline 1 & Which month is the hottest? & Month & $\begin{array}{l}\text { difference, } \\
\text { peak }\end{array}$ & amplitude & attribute & primitive & $1 / 72$ \\
\hline 2 & $\begin{array}{l}\text { Which month is followed by a sudden } \\
\text { change in temperature? }\end{array}$ & Month & $\begin{array}{l}\text { difference, } \\
\text { trend }\end{array}$ & amplitude & implicit attribute & primitive & $1 / 66$ \\
\hline 3 & Which year is the hottest on average? & Year & $\begin{array}{l}\text { difference, } \\
\text { peak }\end{array}$ & amplitude & attribute & composite & $1 / 6$ \\
\hline 4 & Which year has an irregular pattern? & Year & $\begin{array}{l}\text { irregular } \\
\text { fluctuations }\end{array}$ & phase shift & existence, layout & composite & $1 / 6$ \\
\hline 5 & $\begin{array}{l}\text { Which year has most frequent changes } \\
\text { between hot and cold months? }\end{array}$ & Year & $\begin{array}{l}\text { distribution, } \\
\text { seasonality }\end{array}$ & frequency & existence, layout & composite & $1 / 6$ \\
\hline
\end{tabular}

\subsection{Apparatus}

Visual stimuli were created using custom software that was written in $\mathrm{C}++$ in conjunction with Qt. Stimuli were saved as static images and presented to participants using a custom made interface. Experiments were run using Intel $($ dual core PCs running at $2.13 \mathrm{GHz}$, with $2 \mathrm{~GB}$ of RAM and Windows XP Professional. The display was 19" LCD at $1280 \times 1024$ resolution with a 32 bit sRGB color mode. Each monitor was adjusted to have same brightness and same level of contrasts. Participants interacted with the software using a standard mouse at a desk in a dimmed experimental room.

\subsection{Stimuli}

A total of 120 stimuli were used in this study, and they were organized as 5 groups for different tasks. The 24 stimuli were further divided into four levels, each with 6 stimuli. Each stimulus is a $6 \times 12$ image grid as shown in Fig. 4, and it corresponds to a unique temperature dataset. The datasets were designed to represent the temporal distribution of 12 monthly temperature samples from a fictional territorial area spread over a period of 6 years. Temperature datasets were created artificially, and mimicked, as far as possible, real temperature distributions taken from [22]. Our artificial temperature range varied from -40 to +40 degrees.

For each temperature value, a $400 \times 400$ PNG uniform pixel block was created (LO block, see Fig. 5a). Colors were determined based on a white-to-red gradient mapping as shown in Fig. 3a. This mapping has proven to be colorblind friendly in accordance to the guidelines provided in [12]. Color mapping was performed using a two step conversion process: first from a temperature value to a CIE $\mathrm{L}^{*} \mathrm{u}^{*} \mathrm{v}^{*}$ value, and then from an $\mathrm{L}^{*} \mathrm{u}^{*} \mathrm{v}^{*}$ value, via the CIE XYZ color space, to gamma-corrected sRGB value for display on sRGB calibrated screen. For this white-to-red CIE L* $\mathrm{u}^{*} \mathrm{v}^{*}$ color space transformation we used standard correction formula as in [20]. The value of the reference

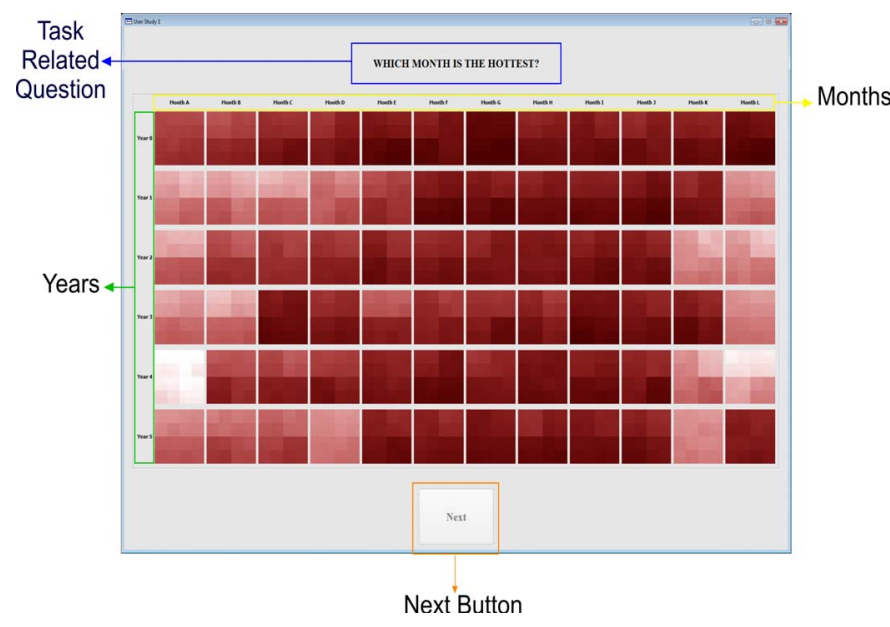

Fig. 4. User Study 1. User Interface Description. white chosen for the present study was the maximum monitor white as in [34]. From each L0 block, three higher resolution blocks at levels 1, 2, and 3 (L1, L2 and L3 blocks, see Fig. 5b-c-d) were created iteratively. These L1, L2 and L3 blocks were generated by using a quadtree, with the L0 block as the root. The nodes of each quadtree at level $L>0$ contained the pixel values for the block at resolution $L$, and these values were computed from values at level $L-1$ using a midpoint displacement algorithm with roughness factor equal to 0.5 . The background color was chosen to convey neutrality in relation to the color information within the grid quadrants.

\subsection{Tasks}

Participants performed five main tasks each probing a specific aspect of the exploratory process typically conducted by scientists. Table 1 summarizes the main design attributes of the five tasks as outlined in Section 4. For each task, it lists the question asked, the unit of response (month/year), the nature of the task demands, the nature of the time series phenomena, the nature of the evaluation performed, the nature of the target response and the overall probability of a correct answer via random guess.

Task 1 involved visual search for a unique target, the hottest month, within the grid. Target months were designed to have at least a $15 \%$ magnitude difference from the next nearest distractor.

Task 2 involved estimating temperature changes between consecutive months, in order to locate the largest such increase across the whole display. Temperature increases always occurred from left to right. Target pairs were designed to have at least a $20 \%$ magnitude difference between each other, compared to $10 \%$ for the nearest distractor pair. In Tasks 1 and 2, users were asked to indicate their response by clicking with the mouse over the target month. The comparison in Task 2 is based on the evaluation of changes between two neighboring blocks. Such changes are not explicitly given, and we hence call such an attribute an implicit attribute. The participants have to carry out two levels of change evaluation, first between two neighbors in each pair, and then between changes taking place in different months and years.

Task 3 required participants to evaluate which year was the "hottest" on average. For this and the remaining 2 tasks, the selection of any month within a row resulted in the selection of the entire row/year.

Both Tasks 4 and 5 required the participants to search for the year with a pattern of behavior not synchronized with the others. Task 4 involved detecting a difference in phase between the signal characterizing the target and all other years. Non-target years were created using sine or cosine functions with a uniform phase shift as their only differentiating feature. Target stimuli were created using functions with non-uniform or opposite phase shifts to the distractors.

Task 5 required users to detect and count temperature transitions within each year. Target years included at least $20 \%$ more transitions than the nearest distractor year. Transition periodicity was inserted into the data in the form of functions with different frequency. Target stimuli were designed to be high frequency sine or cosine functions while distractors followed a normal distribution. 


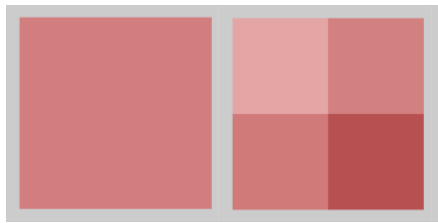

(a) LO block (b) L1 block

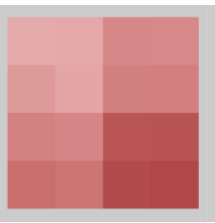

(c) L2 block

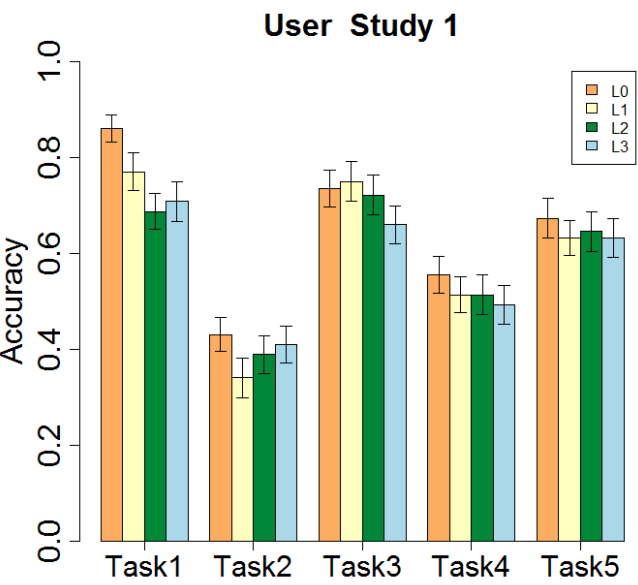

(a) Accuracy

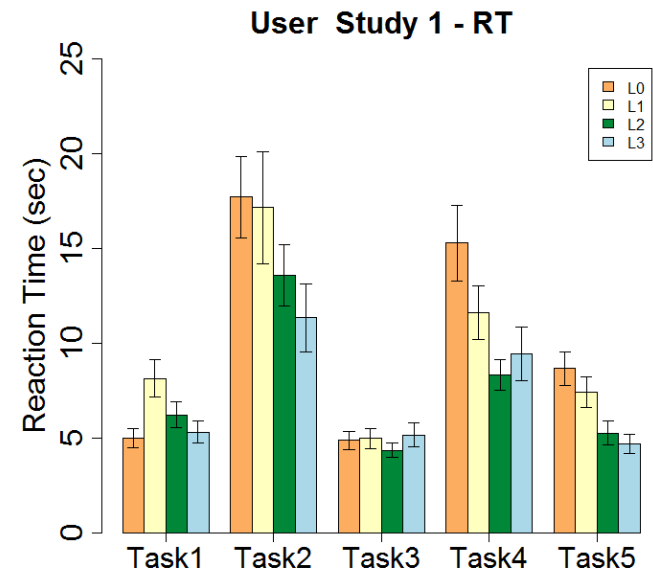

(b) Reaction Time
The experiment began with a brief overview read by the experimenter using a predefined script. Detailed instructions were then given through a self-paced slide presentation. Brief descriptions of the requirements of each task were also provided (at each terminal).

Each participant completed a total of 120 trials, separated into 5 blocks of 24 trials. The 5 tasks were always completed in sequential order, as we wanted to block month and year trials and to avoid confounding task difficulty with initial familiarization with the scenario. For a similar reason, we also controlled the presentation order of block resolution. Within a given task, all trials at level 0 were completed before moving on to level 1, then level 2 and finally level 3. Randomness was introduced at "year" level, rows were randomly swapped between each display to reduce the learning effect.

Specific instructions were given onscreen before each task and 12 practice trials were also completed. At the end of each task, participants took a short break. When all tasks had been completed each participant completed a short debriefing questionnaire and were provided with information about our experimental goals.

\subsection{Results}

Performance in this experiment, as a function of task and block resolution level, is summarized in Fig. 6. There is clearly noticeable variation in performance across tasks, and the magnitude of this variation is more striking than we expected. As shown in Fig. 6a, peak accuracy performance is in Task 1, with $76 \%$ on average across all 4 levels, followed by $72 \%$ for Task 3, 65\% for Task 5, 52\% for Task 4, and conspicuously $39 \%$ for Task 4 . Reaction time patterns (Fig: 6b) show a similar trend show a similar trend where Task 3 leads to fastest average responses, at $4.8 \mathrm{sec}$., followed by Task 1 at $6.2 \mathrm{sec}$., Task 5 at $6.5 \mathrm{sec}$., Task 4 at $11.2 \mathrm{sec}$. and Task 2 at $15.0 \mathrm{sec}$. The impact of block resolution appears to be less clear-cut, with the largest change in performance occurring in Task 1, where uniform patterns gave rise to an accuracy advantage of around $10 \%$ and a speed decrease of several seconds. Overall, the pattern of accuracy and reaction time data show no hint of a speed/accuracy tradeoff.

To explore these patterns in more detail a 5 (Task) $\times 4$ (Level) repeated measures analysis of variance (ANOVA) was used to examine the accuracy and the reaction time data.

For accuracy data, there were main effects of both Task, $\mathrm{F}(4,92)=21.5, \mathrm{p}<0.001$, and Level, $\mathrm{F}(3,69)=5.2, \mathrm{p}<0.001$, and no interaction. To further examine the impact of Task, we computed pairwise comparisons of all means, using Bonferroni correction to adjust for multiple testing. This indicated that performance in both Task 2 and Task 4 were significantly lower than in the other three tasks (all ps $<.05)$ but were not statistically different from each other. No other comparisons were significant. To examine the effect of levels upon each task, we ran separate one-way repeated measures ANOVAs. In Task 1 , there was a main effect of level, $F(3,69)=9.5, p<0.001$. Accuracy at level 0 is consistently better than those at levels 2 and 3, but the difference against Level 1 is not statistically significant. Level 1 stimuli also led to better accuracy than those at level 2, but not compared with Level 3. For the accuracy of other four tasks, there were no other reliable differences.

For reaction time data there were main effects of both Task, $\mathrm{F}(4,92)=29.6, \mathrm{p}<0.001$ and Level, $\mathrm{F}(3,69)=14.1, \mathrm{p}<0.001$, and no interaction. One-way ANOVAs showed a consistent main effect
Fig. 6. User Study 1. Overall accuracy and reaction time for the four levels per task. Error bars show standard error.

of level for Task 1, F(3,69)=10.6, p $<0.001$, with comparison of means indicating that responses to level 1 were slower than all other levels. Task 2 also showed a main effect of level, $F(3,69)=3.3, p<$ 0.05 , which appears to be driven by rapid responses to level 3 stimuli $(\mathrm{p}=.05)$. The Task 4 main effect, $\mathrm{F}(3,69)=6.9, \mathrm{p}<0.001$, is driven by slow responses to level 0 stimuli, although this was only reliably different from level 2 responses. Finally, Task 5 responses were affected by level, $\mathrm{F}(3,69)=18.9, \mathrm{p}<0.001$, with levels 1 and 2 being slower than levels 3 and 4 .

\subsection{Discussion}

The main purpose of Study 1 was to provide an initial assessment as to how the task performance is affected by the nature of tasks and the different levels of block resolution. From Fig. 6, we can observe the difference between different tasks, suggesting different perceptual load because of the task characteristics shown in Table 1. Meanwhile, the cost associated with higher resolutions in all tasks was modest, never exceeding a $6 \%$ increase in errors. In general our users were able to extract monthly or yearly averages of (the increased) resolution.

Results showed a clear per task variation in performances, tasks that required searching for specific trends, either at the month or year level (Tasks 1, 3 and 5) were performed well, those that required processing of change or detection of seasonal variations across the display gave rise to high error rates.

The surprising finding in Study 1 was that the performance of Task 2 , in both accuracy and reaction time, was very poor. We had not 


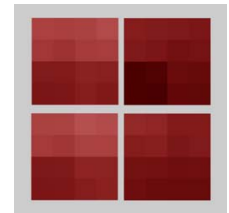

(a) $\mathrm{HH}$

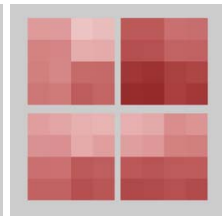

(b) $\mathrm{CC}$

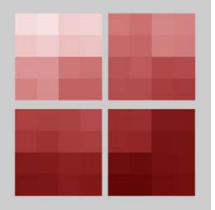

(c) $\mathrm{CH}$

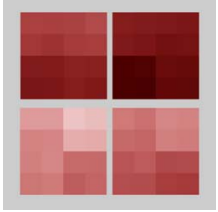

(d) $\mathrm{HC}$

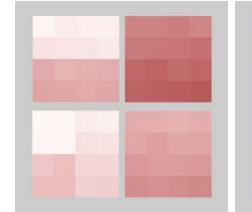

(e) $\mathrm{HH}$

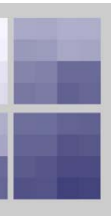

(f) $\mathrm{CC}$

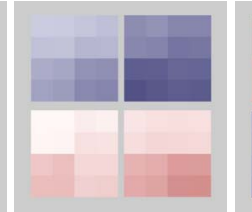

(g) $\mathrm{CH}$

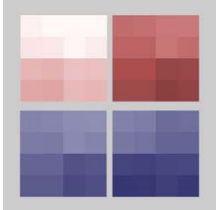

(h) $\mathrm{HC}$
Fig. 7. User Study 2. Examples of stimuli pairs seen on each trial for the four categories in both white-red (a-d) and blue-white-red (e-h) conditions.

expected performance to drop below $40 \%$ correct, though this is still much higher than the chance of a random guess $(1.5 \%)$. Note we cannot compare the perceptual load with tasks 3,4 and 5 directly based on the results in Fig. 6, as they have a much higher chance of a correct answer via random guess $(17 \%)$.

In addition to the empirical results, post-hoc discussions with the users also indicated that this task was particularly difficult. Although we had designed the target change to be at least $20 \%$ larger than the next nearest change in temperature, many users reported that locating the target change was extremely difficult, while others reported identifying multiple possible targets with no way to distinguish between them. Such large variation in performance highlights the relationship between task load and visual characteristics of a display. Therefore we chose Task 1 and Task 2 for further inspection as the two tasks with highest and lowest performance results. In the following studies, we tried to establish whether other aspects of the displays, such as the context or the color spaces, in addition to the change task itself, might be contributing to this pattern of results.

\section{USER STUdY 2}

In order to explore the source of the errors in Task 2 of the previous study, we made a number of design modifications aimed at increasing the diagnostic power of the experimental procedure. Specifically, we removed the search component of the task, focusing more directly on assessment of change, reduced the number of block resolutions levels from four to three, and sampled the color space in a more comprehensive manner. More details in these modifications are provided below.

\subsection{Participants}

There were 21 participants recruited for this study (4 male and 17 female), each took part in this experiment in return for partial course credit or a $£ 5$ book voucher. Students were recruited from the Swansea University community, again from a variety of disciplines. Ages ranged from 18 to 39 (Mean=21.76, $\mathrm{SD}=4.18$ ). Participants were randomly assigned to one of three experimental conditions, with 7 participants in each group. All participants had normal or corrected to normal vision and were not informed about the purpose of the study at the beginning of the session.

\subsection{Apparatus and Stimuli}

Visual stimuli were created using the custom software written in $\mathrm{C}++$, with Qt as graphics library used in Study 1, which mapped a -40 to +40 temperature range into the appropriate color space (see details of each condition below). Stimuli were saved as static images and presented to participants via custom written MATLAB routines using
Psychophysics Toolbox Version 3 (PTB-3) [5]. Presentation was controlled using a Macintosh G5 computer running at $2.1 \mathrm{GHz}$, with 4 GB of RAM and OSX 10.4.2. The monitor was a color-calibrated 21" cinema display (visible area $41 \mathrm{~cm}$ by $30 \mathrm{~cm}$ ) with a resolution of 1024 $\times 768$ pixels and an effective refresh rate of $75 \mathrm{~Hz}$. Participant responses were recorded via a standard keyboard at a desk in a dimmed experimental room.

\subsection{Task Design and Procedure}

On each trial, users were presented with two pairs of images (one pair in the upper part and one pair in the lower part of the screen, see Fig. 7) representing the change in temperature between consecutive months. Users had to indicate which pair contained the greatest increase in temperature by pressing either "T" or "B" for top and bottom respectively.

The size of the target change was randomly selected to be either 12 or 16 degrees. The distractor change was randomly selected to be 4 or 8 degrees. Target pairs had an equal probability of occurring in the upper or lower part of the screen. Trials were organized into four categories, depending on the section of the temperature range that each pair originated from. These categories were labeled hot-hot $(\mathrm{HH})$, cold-cold (CC), hot-cold (HC) and cold-hot $(\mathrm{CH})$. The first member of each Hot pair was randomly selected to be within the range +2 and +12 degrees, and the first member of each Cold pair between -32 and -28 degrees. These constraints were designed to sample the mid regions of each temperature range while avoiding the end points. As before, the block resolution was varied. This time only 3 levels were used and were randomly intermixed rather than blocked. The order of trial presentation was randomly generated on a user-by-user basis.

Participants were assigned to one of three groups; Red-White RGB, Red-White CIE L*u*v* or Blue-White-Red sRGB. Each participant was presented with a total of 480 trials, presented in blocks of 60 , following which the participants were given the option of a short break. In a change to the previous study, the resolution of the images was randomized, as was each of the four conditions (hot-hot, cold-cold, hot-cold, cold-hot), leading to a 3 (conditions) $\times 3$ (block resolution level) $\times 4$ (categories) repeated measures design. All other aspects of the procedure were the same as described in Section 6.5.

\subsection{Condition 1: White-Red in $\operatorname{CIE~} L^{*} \mathbf{u}^{\star} \mathbf{v}^{*}$}

The users in Condition 1 were shown stimuli that were generated using the same color mapping as in Study 1. Our purpose was to see whether the high error rates measure in Task 2 of Study 1 would be replicated, and whether our experimental modifications allowed us to more precisely locate the source of those errors.

\subsubsection{Results}

Performance in this experiment, as a function of color category and block resolution level, is summarized in Fig. 8a and Fig. 8b. When changes had to be evaluated in pairs from the same category, performance was consistently good, exceeding $75 \%$ correct and remaining below RTs of 2 seconds across all resolutions. In both the HH and CC categories, there appears to be a clear performance advantage, in both speed and accuracy, for level 0 representations. When changes across categories had to be evaluated, however, the story is very different. Users appear to have a strong bias to select the pair from the colder category, leading to much slower, chance-level performance in the $\mathrm{HC}$ category. Although performance appears to be excellent in the $\mathrm{CH}$ condition, it seems highly likely that this is an outcome of the general bias to favour the cold pair.

A 4 (pair color category) $\times 3$ (block resolution level) repeated measures ANOVA was used to explore these patterns for both speed and accuracy.

For the accuracy data there were main effects of both Category, $\mathrm{F}(3,15)=40.6, \mathrm{p}<0.001$, and Level, $\mathrm{F}(2,10)=4.6, \mathrm{p}<0.05$, and no interaction. Comparison of means indicated that the HC category was significantly lower than all other categories (all ps $<0.05$, accuracy). No other comparisons were significant. Separate one-way ANOVAs were computed for each category to more fully explore the effect of 

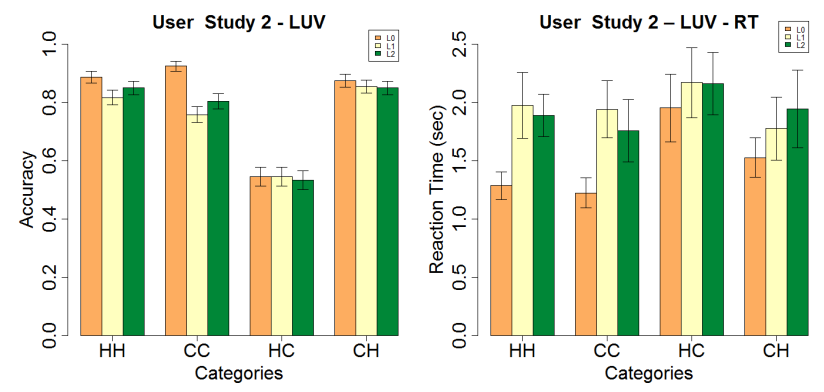

(a) WR, CIE L*u*v*, accuracy

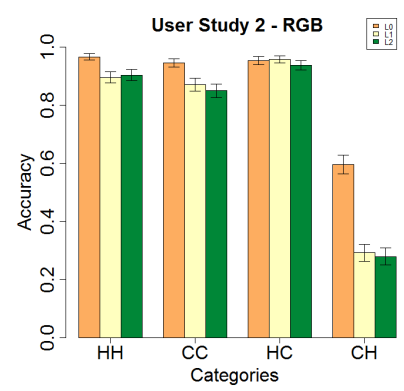

(c) WR, sRGB, accuracy

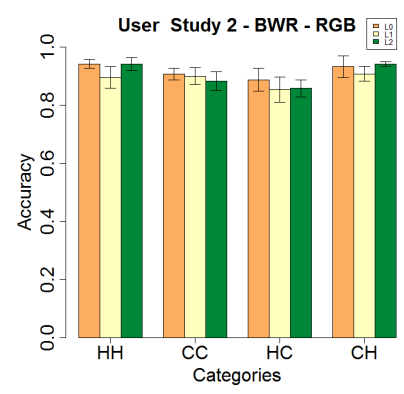

(e) BWR, sRGB, accuracy (b) WR, CIE L* $\mathrm{u}^{*} \mathrm{v}^{*}$, reaction time

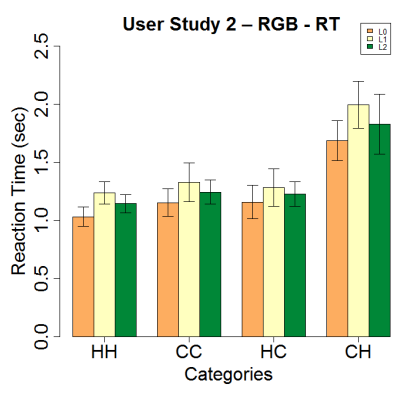

(d) BR, sRGB, reaction time

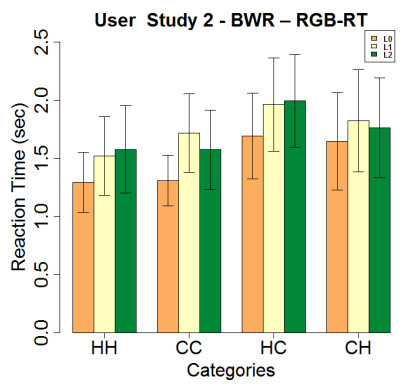

(f) BWR, sRGB, reaction time

Fig. 8. User Study 2. Overall accuracy and reaction time for the 3 levels $\times 4$ conditions of each color space. Three color spaces are White-Red in CIE L* $u^{*} v^{*}$, White-Red in sRGB, and Blue-White-Red in sRGB. Error bars show standard error.

level. This revealed that only the $\mathrm{CC}$ category has a reliable main effect of level, $\mathrm{F}(2,10)=15.6, \mathrm{p}<0.001$. This resulted from performance in level 0 being significantly greater than levels 1 and 2 .

The reaction time data showed main effects of both Category, $\mathrm{F}(3,15)=7.8, \mathrm{p}<0.01$ and Level, $\mathrm{F}(2,10)=8.0, \mathrm{p}<0.01$ and no interaction. Comparison of means showed a significant decrease in performance only between $\mathrm{HC}$ and $\mathrm{CC}$ categories $(\mathrm{p}<0.02)$. No other comparisons were significant. Separate one-way ANOVAs revealed main effects of Level for both the $\mathrm{HH}, \mathrm{F}(2,10)=8.0, \mathrm{p}<0.01$, and CC, $\mathrm{F}(2,10)=10.1, \mathrm{p}<0.01$ reflecting the rapid responses to level 0 stimuli.

\subsubsection{Discussion}

Our goal was to try and localize the cause of the poor performance in evaluating changes during Task 2 of Study 1. The current results indicate errors arise when users need to explicitly compare the magnitude of changes in pairs from different sides of the temperature range. More specifically, there is a bias to judge changes in lower temperature pairs as being greater when compared to a higher temperature pair. If a similar bias were present in our previous study, this may have led users to miss-assign the target change, and even to identify multiple potential targets.

How can we explain this bias? It has been well established that physically equal steps in color space are not perceived as such [30, 34]. The visual systems response to certain color and luminance changes is not linear, but varies according to overall intensity [21]. Psychophysical "laws" such as Weber's Law and Stephen's Law describe neatly how detection varies inversely with the overall intensity of a display.

The standard color mapping and correction we used to create our stimuli attempts to account for these perceptual effects, essentially by amplifying changes when intensity levels are high. That is, steps for each degree of temperature at the lower end of our range are physically larger than steps at the upper end. Theoretically, this should give rise to perceptually equalized steps in color space. Clearly, however, our users place more weight on the overall intensity differences than on the intended "perceptually uniform" color changes.

The effect of block resolution observed in this condition was again similar to that observed in Study 1. Block resolution did not appear to be the source of errors, a significant decrement in performance could be measured only between level 0 and other levels.

\subsection{Condition 2: White-Red in SRGB}

If the errors we have observed in the previous studies originate in our attempts to use a perceptually uniform color space, or at least in our users assigning more weight to intensity differences rather than perceived color changes, can we improve performance by using a physically linear color space? To test this idea, we generated a new set of stimuli using uncorrected sRGB values. In these stimuli, the physical difference in intensity from degree-to-degree is uniform across the whole temperature/color range. All other aspects of the experiment were identical to Condition 1, except data was collected from 7 new users.

\subsubsection{Results}

Performance in this experiment, as a function of color category and block resolution level, is summarized in Fig. 8c and Fig. 8d. It is clear from the figure, that overall performance has not been improved by removing the CIE $\mathrm{L}^{*} \mathrm{u}^{*} \mathrm{v}^{*}$ mapping, rather the location of the bias has shifted. Again, when pairs came from the same temperature range (HH and CC), performance was rapid and near to ceiling. Now though, the slow, error-prone responses have shifted to the $\mathrm{CH}$ category. This indicates that users in this condition have a strong bias to see changes in the warmer part of the range as larger.

The same 4 (pair category) $\times 3$ (block resolution level) repeated measures ANOVA was again used to explore both accuracy and reaction time.

For accuracy, there were main effects of both Category, $\mathrm{F}(3,15)=62.4, \mathrm{p}<0.001$, and Level, $\mathrm{F}(2,10)=46.7, \mathrm{p}<0.001$. There was also a significant interaction between these factors, $F(6,30)=7.6$, $\mathrm{p}<0.001$. This interaction would appear to arise due to the large increase in errors between levels in the $\mathrm{CH}$ category. To further explore the main effect of category, a comparison of all means was conducted. This indicated that performance in the $\mathrm{CH}$ category was significantly worse than in all other categories and that performance in the $\mathrm{HC}$ category was significantly better than both the $\mathrm{CC}$ and the $\mathrm{CH}$ conditions (all ps $<0.05$ ). A similar comparison for the main effect of level indicated that performance with level 0 stimuli was significantly better than with level 1 or 2 stimuli (ps $<0.05$ ). To further examine the interaction between Category and Level, separate one-way ANOVAs were conducted for each color category. This confirmed the presence of a level 0 advantage in accuracy, in all categories except $\mathrm{HC}$ (all Fs > 6.0 , ps $<0.05)$.

For reaction time, there was only a main effect of Category, $\mathrm{F}(3,15)=24.4, \mathrm{p}<0.001$. Means comparison indicated that performance in the $\mathrm{CH}$ category was significantly slower than in any other category. There was a trend for level 0 responses to be faster, although this did not reach significance, $\mathrm{p}=0.09$.

\subsubsection{Discussion}

Rather than reducing the overall rate of errors, the physically uniform color space simply shifted them in a rather predictable manner. That is, 
the tendency of users to over-estimate the changes occurring at the hot end of our temperature range (i.e. when overall stimulus intensity is lower) is precisely the pattern of results that would be predicted based on the non-linear properties of the visual system. Simply ignoring these limitations, would not seem to be an option, at least given the current task. As in the previous condition, a small, but reliable cost of increasing block resolution was again present in this task between level 0 and other levels.

\subsection{Condition 3: Blue-White-Red in SRGB}

In the final condition of this study, we wanted to explore one simple option for reducing the errors seen in conditions 1 and 2. Our idea was to effectively compress the overall range of intensity values by using two color hues instead of one. We re-mapped temperatures lower than zero using a White-Blue scale and temperature above zero using a White-Red scale (see Fig. 3c). This re-mapping, together with the target selection method employed in the previous two studies, ensured that pairs across all four of our color categories were now much more closely matched in terms of overall intensity. Would this modification improve overall performance?

\subsubsection{Results}

The results from this manipulation are shown in Fig. 8e and Fig. 8f. It is immediately obvious that systematic errors have been reduced. Performance in all Categories and across the three levels remained close to ceiling levels. The same 4 (pair category) $\times 3$ (block resolution level) repeated measures ANOVA used in the previous two experiments was applied to both speed and accuracy data. For accuracy there were no main effects, nor interactions. For the reaction time data, there were main effects of both Category, $\mathrm{F}(3,15)=3.4, \mathrm{p}<0.05$, and Level, $\mathrm{F}(2,10)=5.9, \mathrm{p}<0.05$, but no interaction. The Category effect would appear to reflect slightly faster responses for the within category decisions of $\mathrm{HH}$ and $\mathrm{CC}$, although further analysis revealed no significant differences. The effect of Level was also limited to the $\mathrm{HH}, \mathrm{F}(2,10)=4.5, \mathrm{p}<0.05$, and $\mathrm{CC}, \mathrm{F}(2,10)=5.4, \mathrm{p}<0.05$ conditions. Although this pattern would seem to favor faster responses for level 0 stimuli, further analysis could not confirm this pattern.

\subsubsection{Discussion}

Conditions 1 and 2 of this study demonstrated that user responses can sometimes be biased by properties of a color space other than those intended by a given task. It seems likely that performance in Task 2 of Study 1, were also caused by such factors. Although our tasks were aimed at detecting changes in color saturation, observers were highly sensitive to changes in intensity. The results of condition 3 suggest that one possible solution, at least in this specific task, would be to try and compress the range of intensity values, in our case, by introducing a second hue. No block resolution effect in this condition was reported even with near ceiling levels of performance.

\section{USer STUdy 3}

In our final study, we examined more fully the influence of block resolution on the participants' performance. In Studies 1 and 2, there have been small but reliable advantages for the uniform patterns of level 0 versus other levels. In Study 1, this advantage was most apparent for the simple task of identifying the hottest month (i.e., Task 1). Here, we return to this particular task with a slightly modified procedure, to explore whether this advantage is truly robust. The same design framework described in Section 6 was adopted and interface, interaction and apparatus remained unchanged.

\subsection{Participants}

Eleven participants ( 7 female, 4 male) took part in this experiment in return for partial course credit or a $£ 5$ book voucher. Students were recruited from the Swansea University community as in the previous studies. Ages ranged from 18 to 43 (Mean=27.9, SD=6.36). All participants had normal or corrected to normal vision and were not informed about the purpose of the study at the beginning of the session.

\subsection{Stimuli}

Stimuli used in Test 1 were modified for this study. The changes are:

- We kept the three lower levels of resolution but removed the highest level (level 3), since it was found in Study 1 that there is no sufficiently noticeable difference between resolution levels 2 and 3 .

- We randomized the positions of pixel blocks at each level, rather than following a seasonal pattern, as in User Study 1, where the positions of pixels blocks were governed approximately by a cold-to-warm-to-cold annual cycle.

- Stimuli were divided into 4 basic bands of the temperature range, labeled as Very Hot (VH, target chosen from the hot region extreme between $70 \%$ and $90 \%$ of gradient), Hot (HO, target chosen half way of hot region between $50 \%$ and $70 \%$ of gradient), Warm (WA, target chosen close to mid region between $30 \%$ and $50 \%$ of gradient), and Cold (CO, target chosen half way of cold region between $10 \%$ and $25 \%$ of gradient). We avoided to chose target/distractor from the extremes of the gradient to compensate the difficulty of detecting changes in these areas.

Same as for Study 1 the CIE L*U*V color space was used. Target months were still required to have at least a $15 \%$ magnitude difference from the distractors.

\subsection{Procedure}

The experiment followed the same overall procedure detailed in Section 6.5. For the purpose of this study though each participant was presented with a total of 288 trials, presented in blocks of 72, following which the participants were given the option of a short break. The order in which the four temperature bands were shown was also randomized.

\subsection{Results}

Fig. 9 shows the performance summarized as a function of block resolution and temperature band. It is clear that the performance was extremely good, remaining above $90 \%$ in all conditions. There is not much noticeable difference in terms of mean accuracy, though a 4 (temperature band) $\times 3$ (block resolution) repeated measures ANOVA revealed main effects of Level, $\mathrm{F}(2,20)=4.5, \mathrm{p}<0.05$, Band, $\mathrm{F}(3,30)=9.0, \mathrm{p}<0.001$, and their interaction, $\mathrm{F}(6,60)=4.8, \mathrm{p}<0.001$. Further analysis of the main effects revealed that the performance at level $0(\mathrm{M}=97 \%)$ was only marginally better than at either level 1 $(\mathrm{M}=97 \%$; $\mathrm{p}=0.08)$ or level $2(\mathrm{M}=96 \% ; \mathrm{p}=0.08)$. The effect of temperature band related to the fact that the performance in the middle two bands were slightly better than at either the Very Hot or Cold extremes (ps $<0.05$ ). To explore the interaction, we ran separate oneway ANOVAs to examine the pattern of block resolution at each temperature band. This revealed that the only reliable difference occurred in the Cold temperature band, $\mathrm{F}(2,20)=8.0, \mathrm{p}<0.001$, where level 0 performance was approximately $6 \%$ better than at levels 1 and 2 . A similar trend was seen for the Hot temperature band, although this did not reach significance, $F(2,20)=2.9, p=0.08$. Reaction time analysis via 4 (temperature band) $\times 3$ (block resolution) repeated measures ANOVA revealed main effects of Level, $F(2,20)=3.7, p<0.001$ (RT), Band, $F(3,30)=46.4, p<0.001$ no interaction was significant. Separate one-way ANOVAs to examine the pattern of block resolution at each temperature band revealed main effect of Level for all bands but a significant interaction only between level 0 and the other levels ( $\mathrm{p}<$ 0.001 ), with an increase of approximately $30 \%$.

\subsection{Discussion}

The goal of this study was to explore in more detail the impact of resolution observed during Task 1 of Study 1. As part of this study, we also examine the impact of resolution in different color bands. The current results confirm that there may be a small cost when the resolution of pixel blocks increases. However, this effect is a marginal one in terms of mean accuracy. The presence of a level $\times$ temperature band interaction indicates that this impact is influenced by other factors. Specifically, our post-hoc analysis suggests that the effect of level 


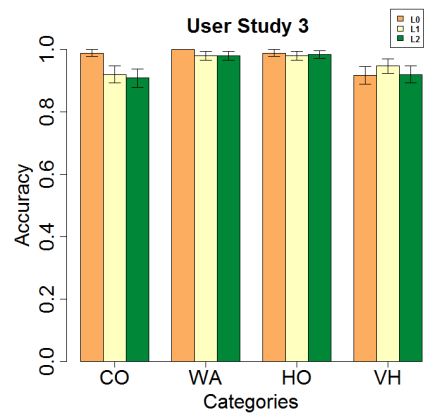

(a) Accuracy

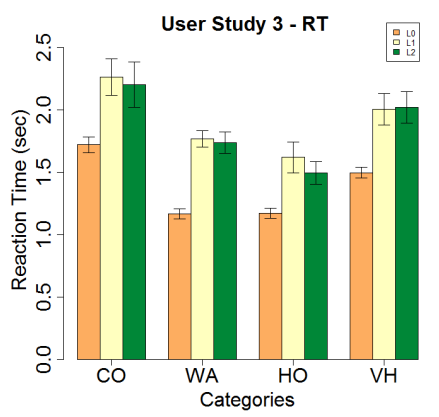

(b) Reaction Time
Fig. 9. User Study 3. Overall accuracy and reaction time for the 3 levels of resolutions $\times 4$ temperature bands (COld, WArm, HOt, Very Hot). Error bars show standard error.

is more observable in the lowest temperature band $(\mathrm{CO})$ that maps to a white-to-pale red color band. The Reaction Time results also support this observation. This indicates that the effect of resolution may depend on the color band, or at least it may be more apparent in some color bands than others.

\section{Discussion Summary}

In this section, we consider the results of three user studies together, and provide our overall observations, each is accompanied by a summary of evidence and the suggested practical impact on the use of pixel-based visualization.

\subsection{The Effect of Resolution of Pixel Blocks}

Observation. Block resolution has a limited impact on the effectiveness of pixel-based visualization, when visualization tasks focus primarily on block-level reasoning.

Evidence. In all three studies the effect of block resolution on accuracy was both numerically small (less than 10\%) and restricted to sub-sets of the data. In Task 1 of Study 1, for example, the results show that it is generally statistically insignificant to differentiate the effect of different levels. Similar results can be found for the same category conditions of Study 2 and the cold temperature band of Study 3 . There thus appears to be no general or consistent accuracy-cost for increasing block resolution. In Study 3 that focuses on Task 1, the observed effect of resolution is marginal in terms of mean accuracy. Reaction time slowing for increasing resolution was observed in some conditions, but again, this was relatively modest and not universal.

Practical Impact. This confirms that pixel-based visualization is a cost-effective, and to a large extent scalable, technique. Although the user studies involved only three or four levels of resolution, the studies have clearly indicated that the impact of levels upon accuracy is expected to be small. There is a noticeable impact on reaction time when changing from level 0 to other levels, but there is no suggestion that the trend of decreasing performance will continue along with the increasing resolution.

Limitation. We focused only on block level reasoning in our user studies, and did not experiment with the spatial reasoning within blocks (e.g., determine how two hottest pixels in different blocks are spatially related). Our finding about the lack of effect of levels should not be generalized to spatial reasoning. Further study is necessary to determine the effect of resolutions for such a task.

\subsection{The Effect of Difference in Tasks Demand}

Observation. There are noticeable effects, depending on the task complexity and cognitive load to perform the task. The order of task difficulty can be expressed as (i) Task $1<$ Task 2 and (ii) Task $1<$ (Task 3, Task 5) < Task 4.

Evidence. User study 1 confirms that the performance of Task 2 and Task 4 are significantly worse than other tasks in terms of both accuracy and reaction time.
The results in Section 6.6 confirm both (i) and (ii). It is not conclusive when comparing Task 2 with Tasks 3, 4 and 5 due to the significant difference in the chance of a correct answer by random guess. Meanwhile, taking the chance into account, Task 1 is much easier than Tasks 3, 4, and 5 .

Practical Impact. Pixel-based visualization is effective for some tasks but not always effective for others. Some tasks can be performed poorly, with a below average accuracy. The poor performance is likely related to the difficulties in quantifying, comparing, and reasoning with changes of pixel blocks. As a guideline, it is recommended that users should be made aware of the possibility of poor accuracy when performing some tasks using pixel-based visualization. As a discipline, we need to develop new visualization techniques to address such difficulties.

Limitation. We have not precisely established the reasons for the poor performance in some tasks. Further study is necessary, especially with a focus on the cognitive load of different tasks.

\subsection{The Effect of Color Mapping}

Observation. In pixel-based visualization, the choice of color space affects the task performance. The CIE $\mathrm{L}^{*} \mathrm{u}^{*} \mathrm{v}^{*}$ color space is not as perceptually uniform as stated in the literature. The White-Red colormap in CIE L* $\mathrm{u}^{*} \mathrm{v}^{*}$ has no clear advantage over that in RGB color space. In pixel-based visualization, using two or more color gradient bands does improve the performance in accuracy, but there is no evidence to suggest any improvement in reaction time.

Evidence. User study 2 confirms these findings. Similar findings on using two color gradient bands were reported previously [34].

Practical Impact. Qualifying changes in any color space is an error-prone task, and should be exercised with caution. For a large data value range coupled with a need for evaluating relatively small changes, multiple color gradient bands can improve the detection of just noticeable difference (jnd) as well as the comparison between changes taking place at different data ranges, provided that in each band the color distance increases significantly between every two neighboring data values.

Limitation. User study 2 only examined Blue-White-Red color map. It is not appropriate to generalize this finding to many bands. In this study, we considered that the white color was the middle point of zero degree, which is the middle point of the data value range as well. Further study is necessary to understand of the effectiveness of multi-color gradient bands for a data value range without semantically meaningful breaking points or with irregularly-spaced breaking points.

\section{Conclusions and Future Work}

In this work we conducted three user studies on aspects of pixel-based visualization, resulting in a quantitative analysis of task and block variations in using such a technique. Our results are most relevant to the users and developers of pixel-based visualization systems, particularly those working with time series analysis (see Section 3).

Based on the four levels examined, we can conclude that pixelbased visualizations do not suffer from noticeable impact due to resolution variation. The perceptual load associated with different tasks can impact upon the performance of the users in a more noticeable manner. For example, Task 2, which reflects a simple day-to-day visualization task in many applications, exhibited a high perceptual load, resulting in poor performance. Study 2 also showed that common judgements, such as estimating change, sometimes interact in unexpected ways with particular display characteristic.

This suggests that users should be provided with careful guidance as to the nature of the errors that could potential be encountered in a specific tasks. Based on the results obtained in this work, we have been discussing our findings with remote sensing researchers. We also hope to further explore our findings as to how the appropriate selection of colormaps can alleviate task problems in more complex practical situations. We also believe that the current empirical work makes a useful first step towards understanding some of the factors that affect the usability of hierarchical pixel-based visualizations. 


\section{REFERENCES}

[1] T. Bachmann. Identification of spatially quantized tachistoscopic images of faces: How many pixels does it take to carry identity? European Journal of Cognitive Psychology, 3:85-103, 1991.

[2] L. D. Bergman, B. E. Rogowitz, and L. A. Treinish. A rule-based tool for assisting colormap selection. In VIS '95: Proceedings of the 6th conference on Visualization '95, page 118, Washington, DC, USA, 1995. IEEE Computer Society.

[3] I. Biederman, A. L. Glass, and E. W. J. Stacy. Searching for objects in real-world scenes. Journal of Experimental Psychology, 97:22-27, 1973.

[4] S. Chiaretti, X. Li, R. Gentleman, A. Vitale, M. Vignetti, F. Mandelli, J. Ritz, and F. R. Gene expression profile of adult t-cell acute lymphocytic leukemia identifies distinct subsets of patients with different response to therapy and survival. Blood, (103):2771-2778, 2004.

[5] M. Chun and Y. Jiang. Top-down attentional guidance based on implicit learning of visual covariation. Psychologicl Science, 10:360-365, 1999.

[6] M. C. F. de Oliveira and H. Levkowitz. From visual data exploration to visual data mining: A survey. IEEE Transactions on Visualization and Computer Graphics, 9:378-394, 2003.

[7] L. S. Exchange, 2010.

[8] D. Field. Relations between the statistics of natural images and the response profiles of cortical cells. Journal of the Optical Society of America, 4:2379-2394, 1987.

[9] R. Gentleman and R. Ihaka. The $\mathrm{r}$ project for statistical computing, 2010.

[10] M. C. Hao, U. Dayal, D. A. Keim, and T. Schreck. Multi-resolution techniques for visual exploration of large time-series data. In EuroVis, pages $27-34,2007$.

[11] L. D. Harmon and B. Julesz. Masking in visual recognition: Effects of two-dimensional filtered noise. science 180:1194, 1973.

[12] M. Harrower and C. A. Brewer. Colorbrewer.org: An online tool for selecting colour schemes for maps. Cartographic Journal, 40(1):27-37, Jun 2003

[13] D. e. a. Hoiem. Putting objects in perspective. Proc. IEEE Comp. Vis. Pattern Recog., 2:2137-2144, 2006.

[14] D. H. Jeong, A. Darvish, K. Najarian, J. Yang, and W. Ribarsky. Interactive visual analysis of time-series microarray data. Vis. Comput., 24(12): 1053-1066, 2008

[15] D. A. Keim. Designing pixel-oriented visualization techniques: Theory and applications. IEEE Transactions on Visualization and Computer Graphics, 6(1):59-78, 2000.

[16] D. A. Keim and H.-P. Kriegel. Visualization techniques for mining large databases: A comparison. IEEE Trans. on Knowl. and Data Eng., 8(6):923-938, 1996.

[17] D. A. Keim, C. Panse, M. Sips, and S. C. North. Pixelmaps: A new visual data mining approach for analyzing large spatial data sets. Data Mining, IEEE International Conference on, 0:565, 2003.

[18] D. A. Keim, C. Panse, M. Sips, and S. C. North. Pixel based visual mining of geo-spatial data. Computers and Graphics, 28(3):327 - 344, September 2004.

[19] T. Lammarsch, W. Aigner, A. Bertone, J. Gartner, E. Mayr, S. Miksch, and M. Smuc. Hierarchical temporal patterns and interactive aggregated views for pixel-based visualizations. In IV '09: Proceedings of the 2009 13th International Conference Information Visualisation, pages 44-50. IEEE Computer Society, 2009.

[20] B. Lindbloom. Cie standard color equations, 2010.

[21] C. G. Mueller. Frequency of seeing functions for intensity discrimination at various levels of adapting intensity. Journal of General Physiology, 34:463-474, 1951.

[22] U. D. of Energy (DOE), 2010.

[23] A. Oliva and A. Torralba. The role of context in object recognition. Trends in Cognitive Sciences, 11:520-527, 2007.

[24] F. Paas, A. Renkl, and J. Sweller. Cognitive load theory: Instructional implications of the interaction between information structures and cognitive architecture. Instructional Science, 32:1-8, 2004.

[25] R. A. Rensink. Visual search for change: A probe into the nature of attentional processing. Visual Cognition, 7:345-376, 2000.

[26] R. A. Rensink. Change detection. Annual Review of Psychology, 53:245277, 2002.

[27] J. Schneidewind, M. Sips, and D. A. Keim. An automated approach for the optimization of pixel-based visualizations. Information Visualization, 6(1):75-88, 2007.

[28] M. H. Shimabukuro, E. F. Flores, M. C. F. de Oliveira, and H. Levkowitz.
Coordinated views to assist exploration of spatio-temporal data: A case study. In CMV '04: Proceedings of the Second International Conference on Coordinated \& Multiple Views in Exploratory Visualization, pages 107-117. IEEE Computer Society, 2004.

[29] P. T. Sowden and P. Schyns. Channel surfing in the visual brain. Trends in Cognitive Sciences, 10(12):538-545, 2006.

[30] S. S. Stevens. Psychophysics of sensory function. Sensory Communication, 1961.

[31] A. Torralba. How many pixels make an image? Proceedings of the IEEE, 94:1948-1962, 2006

[32] A. Torralba and A. Oliva. Statistics of natural images categories. Network Comput. Neural Syst., 14:391-412, 2003.

[33] A. Torralba and P. Sinha. Detecting faces in impoverished images. Technical report, In AI Memo 2001-028, CBCL Memo 208, 2001.

[34] C. Ware. Color sequences for univariate maps: Theory, experiments and principles. IEEE Computer Graphics and Applications, 8:41-49, 1988.

[35] H. R. Wilson, D. K. Mcfarlane, and G. C. Phillips. Spatial-frequency tuning of orientation selective units estimated by oblique masking. Vision Research, 23(9):873-882, 1983.

[36] J. Wolfe and T. Horowitz. What attributes guide the deployment of visual attention and how do they do it? Nature Reviews Neuroscience, 5:1-7, 2004.

[37] B. Yost, Y. Haciahmetoglu, and C. North. Beyond visual acuity: the perceptual scalability of information visualizations for large displays. In CHI '07: Proceedings of the SIGCHI conference on Human factors in computing systems, pages 101-110, New York, NY, USA, 2007. ACM.

[38] H. Ziegler, T. Nietzschmann, and D. A. Keim. Visual analytics on the financial market: Pixel-based analysis and comparison of long-term investments. Information Visualisation, International Conference on, 0:287295, 2008. 